\title{
Three-Arm Randomized Study of Two Cisplatin-Based Regimens and Paclitaxel Plus Gemcitabine in Advanced Non-Small-Cell Lung Cancer: A Phase III Trial of the European Organization for Research and Treatment of Cancer Lung Cancer Group-EORTC 08975
}

\author{
By Egbert F. Smit, Jan P.A.M. van Meerbeeck, Pilar Lianes, Channa Debruyne, Catherine Legrand, Franz Schramel, Hans Smit, \\ Rabab Gaafar, Bonne Biesma, Chris Manegold, Niels Neymark, and Giuseppe Giaccone
}

\begin{abstract}
Purpose: To compare the therapeutic efficacy of paclitaxel plus cisplatin (arm A) versus gemcitabine plus cisplatin (arm B) and arm A versus paclitaxel plus gemcitabine (arm C) in chemotherapy-naive patients with advanced nonsmall-cell lung cancer (NSCLC).

Materials and Methods: Patients were randomly assigned to receive either paclitaxel $175 \mathrm{mg} / \mathrm{m}^{2}$ (3-hour infusion, day 1 ) or gemcitabine $1,250 \mathrm{mg} / \mathrm{m}^{2}$ (days 1 and 8) both combined with cisplatin $80 \mathrm{mg} / \mathrm{m}^{2}$ (day 1 ) or paclitaxel $175 \mathrm{mg} / \mathrm{m}^{2}$ (3-hour infusion, day 1) combined with gemcitabine $1,250 \mathrm{mg} / \mathrm{m}^{2}$ (days 1 and 8 ). Primary end point was comparison of overall survival for $B$ versus $A$ and $C$ versus $A$. Secondary end points included response rate and duration, progression-free survival, toxicities, quality of life [QoL], and cost of treatment.
\end{abstract}

Results: Four hundred eighty patients (arm A, 159; arm B, 160; arm C, 161 patients) were enrolled; all baseline

$I^{\mathrm{N}}$ $\mathrm{N}$ EUROPE, lung cancer remains the first cause of cancerrelated death in men and the fourth most common among women. ${ }^{1}$ Approximately $85 \%$ of lung cancers are non-small-cell lung cancers (NSCLCs). ${ }^{2}$ The distressingly low cure rate for NSCLC (approximately 15\% 5-year survival) can be attributed to the high rate of unresectable disease at presentation and the inability of systemic therapy to cure metastatic disease. For patients with advanced NSCLC, treatment with chemotherapy confers a modest survival benefit ${ }^{3-5}$ and short-lived improvement of quality of life $(\mathrm{QoL})$ when compared with radiotherapy alone or best supportive care. ${ }^{5-7}$ In phase III trials, combinations of cisplatin and one of the new active agents (ie, taxanes [paclitaxel and docetaxel], gemcitabine, vinorelbine, and irinotecan) have produced superior therapeutic results compared with cisplatin alone ${ }^{8,9}$ and older cisplatin-based regimens. ${ }^{10-13}$ Moreover, some of the new regimens are cost-effective. ${ }^{14-16}$ The combination of cisplatin and gemcitabine is one of the most active newer regimens. ${ }^{17}$ Despite modern antiemetic and hydration regimens, cisplatin has substantial side effects that limit its use. ${ }^{18,19}$ There are several ways to circumvent cisplatin-induced toxicities, including omitting cisplatin and replacing it with a cytotoxic drug with similar activity. One such regimen, paclitaxel plus gemcitabine, has been shown in a phase II study to produce a major response rate of $24 \%$ with acceptable toxicity. ${ }^{20}$ On the basis of these considerations, the European Organization for Research and Treatment of Cancer (EORTC) Lung Cancer Group conducted a randomized, phase III characteristics were balanced. Median survival times were as follows: arm A, 8.1 months; arm B, 8.9 months; arm C, 6.7 months. Response rates were $31.8 \%$ for $\operatorname{arm} A, 36.6 \%$ for arm $B$, and $27.7 \%$ for arm C. Other than myelosuppression ( $B \vee A, P<.005)$, no statistically or clinically significant differences were observed for secondary end points. The average treatment costs were $25 \%$ higher in arm C as compared with arms $A$ and $B$.

Conclusion: Gemcitabine plus cisplatin and paclitaxel plus gemcitabine do not increase overall survival in patients with advanced NSCLC as compared with paclitaxel plus cisplatin. Treatment was well tolerated, and most QoL parameters were similar, but costs associated with the nonplatinum arm were highest.

J Clin Oncol 21:3909-3917. ๑ 2003 by American Society of Clinical Oncology.

study to compare the best arm of our previous phase III study in patients with advanced NSCLC, ie, cisplatin plus paclitaxel, ${ }^{11}$ with cisplatin-gemcitabine and paclitaxel-gemcitabine combinations in chemotherapy-naive patients with advanced NSCLC.

From the Departments of Pulmonology and Medical Oncology, Vrije Universiteit Medical Center, Amsterdam; Department of Pulmonology, Erasmus Medical Centre, Rotterdam; Department of Pulmonology, St Antonius Ziekenhuis, Nieuwegein; Department of Pulmonology, Rijnstate Hospital, Arnhem; and Bosch, s'Hertogenbosch, the Netherlands; Department of Medical Oncology, Matero, Spain; European Organization for Research and Treatment of Cancer Data Center, Brussels, Belgium; National Cancer Institute, Cairo, Egypt; and Thoraxklinik, Heidelberg, Germany.

Submitted March 31, 2003; accepted August 9, 2003.

Supported by grant Nos. 2U10 CA11488-28, 5U10 CA11488-29, and 5 U10 CA11488-30 from the National Cancer Institute, Bethesda, MD.

Presented in part at the Annual Meeting of the American Society of Clinical Oncology, San Francisco, CA, 2001, and the European Conference on Clinical Oncology, Lisbon, Portugal, 2001.

The contents of this article are solely the responsibility of the authors and do not necessarily represent the official views of the National Cancer Institute.

Authors' disclosures of potential conflicts of interest are found at the end of this article.

Address reprint requests to E.F. Smit, MD, PhD, Department of Pulmonology, Martini Hospital, PO Box 30033,9700 RM Groningen, the Netherlands; e-mail: e.f.smit@mzh.nl.

(C) 2003 by American Society of Clinical Oncology.

0732-183X/03/2121-3909/\$20.00 


\section{MATERIALS AND METHODS}

\section{Patients}

Patients with histologically or cytologically confirmed NSCLC stage IIIB (caused by malignant pleural effusion or supraclavicular lymph nodes only) and stage IV disease according to the revised staging system of the American Joint Committee on Cancer $^{21}$ were entered onto the study. Additional eligibility criteria included age between 18 and 76 years, WHO performance status $(\mathrm{PS}) \leq 2$, measurable disease, no previous chemotherapy with the exception of prior neoadjuvant or adjuvant chemotherapy that ended more than 1 year before entry, and adequate hematologic, renal, and hepatic function. Previous radiotherapy was allowed provided that an interval of at least 4 weeks had elapsed and the radiotherapy field did not include all measurable lesions used as target lesion. Patients with preexisting brain metastases or leptomeningeal disease who were treated with radiotherapy, stable without medications (eg, corticosteroids), and asymptomatic were eligible The study was approved by the EORTC Protocol Review Committee and all ethics committees of the participating institutions. Written informed consent had to be obtained from all patients and documented according to national regulatory requirements and to the local institution rules. In the course of the trial, we discovered that the written informed consent could not be documented for 33 patients $(6.9 \%)$ included in the trial. Although we could not retrieve the documentation of the informed consent, all these patients were checked on availability of the other source data. For all these patients, the responsible investigator has stated that he/she fully informed the patient orally on all aspects of the trial and certifies that each patient agreed to participate in the trial.

At study entry, the following investigations were performed: full history and physical examination, complete blood cell count and differential, chemistries, creatinine clearance, ECG, and chest x-ray. All investigations were repeated before every cycle. Computed tomography scans and ultrasound were performed to document disease extent optimally and to evaluate response to treatment according to WHO guidelines. ${ }^{22}$ Toxicity was scored according to the National Cancer Institute common toxicity criteria scale. ${ }^{23}$

\section{Therapy}

Patients were randomly assigned to receive paclitaxel $175 \mathrm{mg} / \mathrm{m}^{2}$ on day 1 followed by cisplatin $80 \mathrm{mg} / \mathrm{m}^{2}$ on day 1 (regimen A), gemcitabine 1,250 $\mathrm{mg} / \mathrm{m}^{2}$ on days 1 and 8 and cisplatin $80 \mathrm{mg} / \mathrm{m}^{2}$ on day 1 after gemcitabine (regimen B), or paclitaxel $175 \mathrm{mg} / \mathrm{m}^{2}$ on day 1 followed by gemcitabine $1,250 \mathrm{mg} / \mathrm{m}^{2}$ on days 1 and 8 (regimen $\mathrm{C}$ ). All treatment cycles were repeated every 3 weeks. Cisplatin was dissolved in $500 \mathrm{~mL}$ of normal saline or $5 \%$ dextrose and infused along a program of forced diuresis that included at least $2 \mathrm{~L}$ of fluids. Paclitaxel was dissolved in $500 \mathrm{~mL}$ of normal saline or $5 \%$ glucose and administered as a 3-hour intravenous infusion with prophylactic medication to prevent hypersensitivity reactions. Gemcitabine was dissolved in $500 \mathrm{~mL}$ of normal saline and administered as a 30-minute intravenous infusion. Prophylactic antiemetics during and after cisplatin administration typically consisted of ondansetron and dexamethasone. Treatment was continued for at least two cycles unless this was clearly not in the patient's best interest. Treatment was always interrupted in case of intolerable toxicity, patient refusal, or disease progression. Responding patients received a maximum of six cycles. Dose adjustments and delays for toxicity were defined as per protocol.

\section{QoL Assessment}

QoL was evaluated in a longitudinal design in all randomly assigned patients. QoL assessments were performed at baseline, at the end of each cycle of treatment, every 6 weeks after the end of treatment until progression of the disease (PD), at $\mathrm{PD}$, and thereafter every 3 months until death using the EORTC QoL core questionnaire (QLQ-C30, version 3.0) in conjunction with the EORTC lung module (LC-13).

\section{Health Economics}

The medical resource data collected comprised primary chemotherapy, hospital admissions (overnight stays or days spent in day clinics), consulta- tions with cancer specialists and family doctors, premedication before administration of paclitaxel, antiemetics, cytotoxic agents, use of blood transfusions, and second-line therapy. Because the large majority of the patients in the trial $(77 \%)$ were recruited by hospitals in the Netherlands, 2002 national tariffs for the Dutch health insurance system were used as unit prices for the determination of costs.

\section{Statistics}

Randomization was performed centrally by the EORTC Data Center after stratification for PS (0 to $1 v 2$ ), stage of disease (IIIB $v$ IV), and institute, using the minimization technique. ${ }^{24}$ The primary end point was the pairwise comparison of overall survival between each of the two experimental arms and the control arm (regimen $\mathrm{B} v \mathrm{~A}$ and $\mathrm{C} v \mathrm{~A}$ ); secondary end points included response rate, duration of response, progression-free survival, toxicities, QoL, and cost of treatment. Duration of survival and progressionfree survival were calculated from the date of randomization. For the responders, duration of response was measured from the date of start of treatment to the date of objective PD; patients were censored if new treatment was started before PD. Assuming a median survival in the control arm of approximately 8 months, ${ }^{13}$ a total of 369 deaths were necessary to detect an absolute increase in median survival of 4 months with a two-sided type I error of 0.02 (to keep the overall type I error of 0.05 ) and a power of $80 \%$. $^{25}$ Assuming a 36-month duration of recruitment and another 12 months of follow-up, 450 patients (150 to each treatment arm) needed to be randomly assigned to achieve these statistical requirements. An interim analysis was scheduled after 60 deaths and was submitted to an independent data monitoring committee. The predefined criteria for considering trial closure were a response rate of less than $25 \%$ in one of the treatment arms or association of a treatment arm with excessive toxicity. For the time-toevent end points, the decision rule was based on alpha-spending function ${ }^{26}$ using an O'Brian-Fleming boundary. ${ }^{27}$

All analyses, except for response rate, were performed on all randomly assigned patients according to the intent-to-treat principle. Response rate analysis was based on eligible patients only. Overall survival curves and progression-free survival curves were estimated by the Kaplan-Meier technique ${ }^{28}$ and pairwise differences for time-to-event end points were assessed using the log-rank test $\mathrm{t}^{29}$ at 0.02 two-sided $\alpha$ level. To adjust for confounding variables, the Cox proportional hazards model with backward variable selection procedure was used. ${ }^{30}$ The multivariate model was based on the following factors of possible prognostic value: stage (IIIB $v$ IV), PS (0 to 1 $v 2$ ), histologic subtype (squamous $v$ nonsquamous), sex, and treatment arm. Pairwise comparisons of response rates were performed using a Cochran Mantel-Haenszel test at .02 two-sided $\alpha$ level. Pairwise comparisons of the rates of grade 3/4 toxicity between the standard arm and each of the experimental arms were performed using a Fisher's exact test at .02 two-sided $\alpha$ level. Reported $P$ values concerning differences in toxicity should be interpreted with caution.

Data on QoL were scored according to the algorithm described in the EORTC QLQ-C30 scoring manual. ${ }^{31}$ Nonoverlapping windows were constructed to assign the QoL forms received to one of the assessment points. Data were analyzed using descriptive statistics for the subscales for each study arm at each of the assessment points. A mixed model was applied using a one-step autoregressive covariance structure to investigate pairwise comparisons of changes in QoL score over time. All tests were performed two-sided at a significance level fixed at $\alpha 5 \%$. Statistically significant changes of $\geq 10$ effect points were defined as clinically significant. ${ }^{32}$

\section{RESULTS}

Between August 1998 and July 2000, 480 patients were randomly assigned from 29 institutions. The outline of the trial is provided in Figure 1. Patient characteristics (Table 1) were well balanced among the three treatment arms; the majority of patients had a good PS, adenocarcinoma, and metastatic disease. The number of cycles and relative dose-intensity administered in 


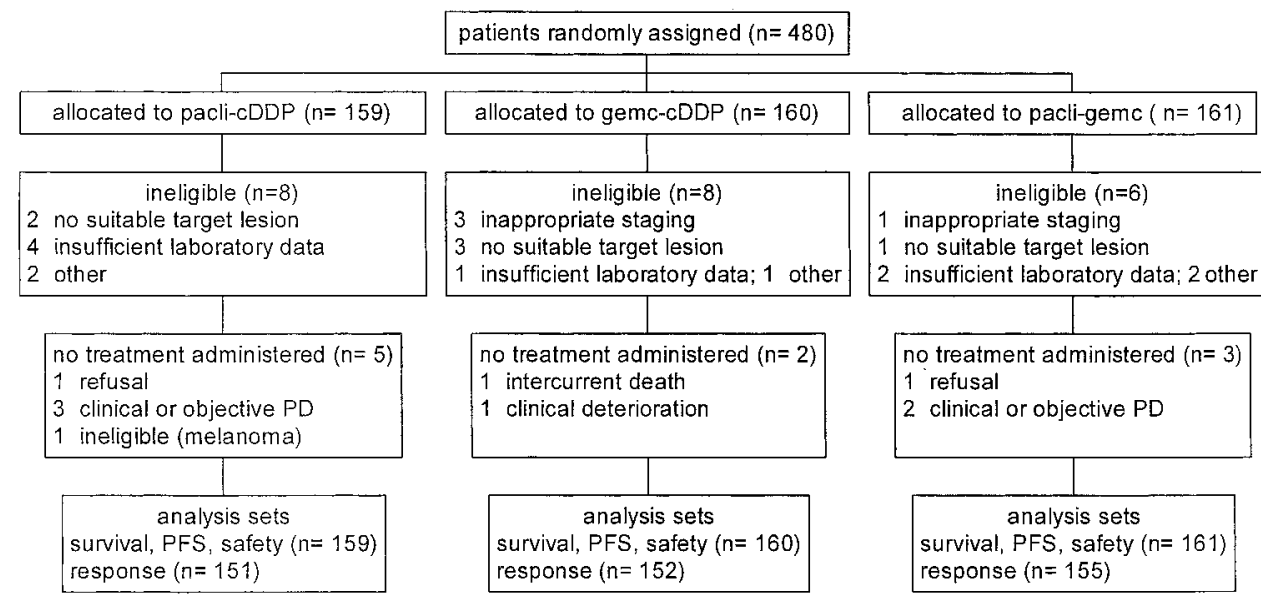

Fig 1. Outline of the study. Pacli, paclitaxel; cDDP, cisplatin; gemc, gemcitabine; $\mathrm{PD}$, progression of disease; PFS, progression-free survival.

the three treatment arms are provided in Table 2. The median number of cycles was lower in arm $\mathrm{C}$ (four cycles) as compared with arms A and B (five cycles). Drug exposure, expressed as relative dose-intensity, was not different between the treatment arms.

\section{Survival and Progression-Free Survival}

After a median follow-up of 28 months, 439 patients (91.5\%) had died: 145 in arm A, 143 in arm B, and 151 in arm C. In 391 $(89.1 \%)$ of these patients, the cause of death was tumor progres-

Table 1. Patient Characteristics

\begin{tabular}{|c|c|c|c|c|c|c|}
\hline \multirow[b]{2}{*}{ Characteristic } & \multicolumn{2}{|c|}{$\operatorname{Arm~A}(n=159)$} & \multicolumn{2}{|c|}{$\operatorname{Arm~B}(n=160)$} & \multicolumn{2}{|c|}{$\operatorname{Arm} C(n=161)$} \\
\hline & No. & $\%$ & No. & $\%$ & No. & $\%$ \\
\hline Ineligible & 8 & 5.0 & 8 & 5.0 & 6 & 3.7 \\
\hline \multicolumn{7}{|l|}{ Age, years } \\
\hline Median & \multicolumn{2}{|c|}{57} & \multicolumn{2}{|c|}{57} & \multicolumn{2}{|c|}{56} \\
\hline Range & \multicolumn{2}{|c|}{$27-75$} & \multicolumn{2}{|c|}{$28-75$} & \multicolumn{2}{|c|}{$31-75$} \\
\hline \multicolumn{7}{|l|}{ Sex } \\
\hline Male & 95 & 59.7 & 113 & 70.6 & 110 & 68.3 \\
\hline Female & 64 & 40.3 & 47 & 29.4 & 51 & 31.7 \\
\hline \multicolumn{7}{|l|}{ WHO PS } \\
\hline 0 & 35 & 22.0 & 40 & 25.0 & 38 & 23.6 \\
\hline 1 & 105 & 66.0 & 102 & 63.8 & 104 & 64.6 \\
\hline 2 & 19 & 11.9 & 18 & 11.3 & 19 & 11.8 \\
\hline \multicolumn{7}{|l|}{ Histology } \\
\hline Squamous & 30 & 18.9 & 41 & 25.6 & 35 & 21.7 \\
\hline Adenocarcinoma & 64 & 40.3 & 73 & 45.6 & 64 & 39.8 \\
\hline Large cell & 63 & 39.6 & 40 & 25.0 & 54 & 33.5 \\
\hline Other & 2 & 1.2 & 5 & 3.1 & 7 & 4.3 \\
\hline Unknown & 0 & 0 & 1 & 0.6 & 1 & 0.6 \\
\hline \multicolumn{7}{|l|}{ Stage } \\
\hline$I I I B$ & 29 & 18.2 & 33 & 20.6 & 29 & 18.0 \\
\hline IV & 130 & 81.8 & 126 & 78.8 & 132 & 82.0 \\
\hline Other & & & 1 & 0.6 & & \\
\hline \multicolumn{7}{|l|}{ Prior surgery } \\
\hline No & 130 & 81.8 & 140 & 87.5 & 128 & 79.5 \\
\hline Yes & 23 & 14.5 & 17 & 10.6 & 24 & 14.9 \\
\hline Explorative thoracotomy & 5 & 3.1 & 1 & 0.6 & 7 & 4.3 \\
\hline Other & 1 & 0.6 & 2 & 1.3 & 2 & 1.2 \\
\hline \multicolumn{7}{|l|}{ Prior radiotherapy } \\
\hline No & 129 & 81.1 & 137 & 85.6 & 132 & 82.0 \\
\hline Yes & 30 & 18.9 & 23 & 14.4 & 29 & 18.0 \\
\hline \multicolumn{7}{|l|}{ Prior chemotherapy } \\
\hline No & 157 & 98.7 & 159 & 99.4 & 154 & 95.7 \\
\hline Yes* & 2 & 1.3 & 1 & 0.6 & 7 & 4.3 \\
\hline
\end{tabular}

Abbreviation: PS, performance status.

${ }^{*}$ (Neo)adjuvant chemotherapy ( $\geq 1$ year before entry). 
Table 2. Chemotherapy Administered

\begin{tabular}{|c|c|c|c|c|c|c|}
\hline & \multicolumn{2}{|c|}{$\operatorname{Arm~A}(\mathrm{n}=159)$} & \multicolumn{2}{|c|}{$\operatorname{Arm~B}(n=160)$} & \multicolumn{2}{|c|}{$\operatorname{Arm} C(n=161)$} \\
\hline & No. & $\%$ & No. & $\%$ & No. & $\%$ \\
\hline \multicolumn{7}{|l|}{ No. of cycles } \\
\hline 0 & 5 & 3.1 & 2 & 1.3 & 3 & 1.9 \\
\hline 1 & 15 & 9.4 & 10 & 6.3 & 19 & 11.8 \\
\hline 2 & 30 & 18.9 & 27 & 16.9 & 34 & 21.1 \\
\hline 3 & 10 & 6.3 & 11 & 6.9 & 9 & 5.6 \\
\hline 4 & 18 & 11.3 & 20 & 12.5 & 24 & 14.9 \\
\hline 5 & 9 & 5.7 & 10 & 6.3 & 8 & 5.0 \\
\hline 6 & 72 & 45.3 & 80 & 50.0 & 64 & 39.8 \\
\hline Median No. of cycles & 5 & & 5 & & 4 & \\
\hline \multicolumn{7}{|l|}{ RDI paclitaxel, \% } \\
\hline Median & \multicolumn{2}{|c|}{100} & & & \multicolumn{2}{|c|}{100.5} \\
\hline Range & \multicolumn{2}{|c|}{$43-109.5$} & & & \multicolumn{2}{|c|}{ 6.8-109.5 } \\
\hline \multicolumn{7}{|l|}{ RDI gemcitabine, \% } \\
\hline Median & & & \multicolumn{2}{|c|}{94.7} & \multicolumn{2}{|c|}{98.6} \\
\hline Range & & & \multicolumn{2}{|c|}{$35-105$} & \multicolumn{2}{|c|}{$14.6-107.4$} \\
\hline \multicolumn{7}{|l|}{ RDI cisplatin, \% } \\
\hline Median & \multicolumn{2}{|c|}{99.9} & \multicolumn{2}{|c|}{96.4} & & \\
\hline Range & \multicolumn{2}{|c|}{$16.9-107.6$} & \multicolumn{2}{|c|}{ 15.9-106.4 } & & \\
\hline
\end{tabular}

sion. Tables 3 and 4 summarize treatment outcome. There were no statistically significant differences in survival times between the reference arm and the two experimental arms. Progressionfree survival was not different between treatment arms, but there was a strong trend for shorter progression-free survival in arm C. Figures 2 and 3 depict the overall survival and progression-free survival by treatment arm.

Multivariate analysis for survival retained only PS 2 as a significant negative prognostic factor for survival; patients with WHO PS 0 to 1 had a median survival of 8.5 months, whereas those with WHO PS 2 had a median survival of 3.3 months $(P<.0001)$.

\section{Tumor Response}

Only one complete response (arm B) was observed (Table 4). The partial response rate was $32 \%$ in arm A, 37\% in arm B, and $28 \%$ in arm C ( $P$ was not significant for both comparisons). Duration of response was similar between arms A and B and showed a strong trend for shorter duration for arm $\mathrm{C}$ as compared with the reference arm.

\section{Toxicity}

There were twelve toxic deaths: four in arm A (2.5\%), two in arm B (1.2\%), and six in arm C (3.7\%). Of these patients, nine had PS 1 at randomization and three had PS 0.

Grade 3 and 4 toxicities that occurred in at least $5 \%$ of patients are listed in Table 5. Hematologic toxicity was the most frequent side effect in all three arms. Nine patients (two in arm A, four in arm B, and three in arm C) developed grade 3 febrile neutropenia, whereas neutropenia grade 3 or 4 occurred in $34.0 \%$ of patients in arm A, 43.1\% in arm B, and $30.4 \%$ in $\operatorname{arm~C~}(P=\operatorname{not}$ significant). Grade 3 and 4 thrombocytopenia occurred more frequently in arm B (36.3\%) as compared with arm A (1.3\%; A $v \mathrm{~B}, P<.0001)$. Anemia grade 3 and 4 was also more frequently observed in arm B (11.9\%) as compared with arm A (3.1\%; A v $\mathrm{B}, P=.0048)$. This is reflected in the proportion of patients with hemorrhagic episodes $(6.4 \%$ in arm A and $15.8 \%$ in arm B; A v $\mathrm{B}, P=.0208)$ and transfusions $(23.9 \%$ in arm $\mathrm{A}$ and $43.7 \%$ in arm B; A $v$ B, $P=.0002$ ), $95 \%$ of which were RBC transfusions. No statistically significant differences for the comparison be-

Table 3. Survival and Progression-Free Survival

\begin{tabular}{|c|c|c|c|c|}
\hline & $\operatorname{Arm~A}(n=151)$ & $\operatorname{Arm~B~}(n=152)$ & $\operatorname{Arm} C(n=155)$ & $P$ \\
\hline \multicolumn{5}{|c|}{ Survival, months } \\
\hline Median & 8.1 & 8.9 & 6.7 & $A \vee B, .668$ \\
\hline $95 \% \mathrm{Cl}$ & 6.2 to 9.9 & 7.8 to 10.5 & 5.9 to 7.6 & $A \vee C, .108$ \\
\hline \multicolumn{5}{|c|}{ 1-Year survival, \% } \\
\hline Median & 35.9 & 33.1 & 26.7 & \\
\hline $95 \% \mathrm{Cl}$ & 28.4 to 43.3 & 25.8 to 40.4 & 19.9 to 33.6 & \\
\hline \multicolumn{5}{|c|}{ PFS, months } \\
\hline Median & 4.2 & 5.1 & 3.5 & $A \vee B, .339$ \\
\hline $95 \% \mathrm{Cl}$ & 3.2 to 4.5 & 4.5 to 5.7 & 2.9 to 4.3 & $A \vee C, .044$ \\
\hline
\end{tabular}

Abbreviation: PFS, progression-free survival. 
Table 4. Response and Response Duration (eligible patients)

\begin{tabular}{|c|c|c|c|c|c|c|c|}
\hline \multirow[b]{2}{*}{ Variable } & \multicolumn{2}{|c|}{$\operatorname{Arm~A}(n=151)$} & \multicolumn{2}{|c|}{$\operatorname{Arm~B~}(n=152)$} & \multicolumn{2}{|c|}{$\operatorname{Arm} C(n=155)$} & \multirow[b]{2}{*}{$P$} \\
\hline & No. & $\%$ & No. & $\%$ & No. & $\%$ & \\
\hline Complete response & 0 & & 1 & 0.7 & 0 & & \\
\hline Partial response & 48 & 31.8 & 55 & 36.2 & 43 & 27.7 & \\
\hline No change & 52 & 34.4 & 56 & 36.8 & 55 & 35.5 & \\
\hline Progressive disease & 36 & 23.8 & 25 & 16.4 & 35 & 22.6 & \\
\hline Early deaths & 8 & 5.3 & 7 & 4.6 & 11 & 7.1 & \\
\hline Not assessable & 7 & 4.6 & 8 & 5.3 & 11 & 7.1 & \\
\hline \multicolumn{8}{|l|}{ Response rate, \% } \\
\hline Median & \multicolumn{2}{|c|}{31.8} & \multicolumn{2}{|c|}{36.8} & \multicolumn{2}{|c|}{27.7} & $A v B, .355$ \\
\hline $95 \% \mathrm{Cl}$ & \multicolumn{2}{|c|}{24.4 to 39.2} & \multicolumn{2}{|c|}{29.2 to 44.5} & \multicolumn{2}{|c|}{20.7 to 34.8} & $A \vee C, .440$ \\
\hline \multicolumn{8}{|c|}{ Response duration, ${ }^{*}$ months } \\
\hline Median & \multicolumn{2}{|c|}{8.0} & \multicolumn{2}{|c|}{7.4} & \multicolumn{2}{|c|}{6.9} & $A v B, .363$ \\
\hline $95 \% \mathrm{Cl}$ & \multicolumn{2}{|c|}{6.4 to 11.0} & \multicolumn{2}{|c|}{6.4 to 9.5} & \multicolumn{2}{|c|}{5.7 to 7.5} & $A \vee C, .040$ \\
\hline
\end{tabular}

*Responding patients only.

tween arm A and C with regard to these toxicities were found. No significant differences were observed between the treatment arms for any of the other grade 3 and 4 toxicities, including anorexia, nausea, and vomiting.

\section{Second-Line Therapies}

Of all patients entered onto the trial, 278 (57.9\%) received at least one form of further antitumoral treatment after PD and were distributed similarly across the treatment arms $(57.7 \%$ in arm A, $59.4 \%$ in arm B, and $59.6 \%$ in arm C). The study protocol did not give any recommendations on second-line therapy. More than $60 \%$ of these patients received radiotherapy, and a third received chemotherapy first as second-line therapy. Again, type of first treatment at PD was similarly distributed among the treatment arms. Of note, of the 28 patients randomly assigned to arm $\mathrm{C}$ who received second-line chemotherapy, 24 patients were treated with a platinum combination.

\section{QoL Analysis}

Compliance at baseline and throughout the active treatment period was greater than $60 \%$ but decreased dramatically at cycle

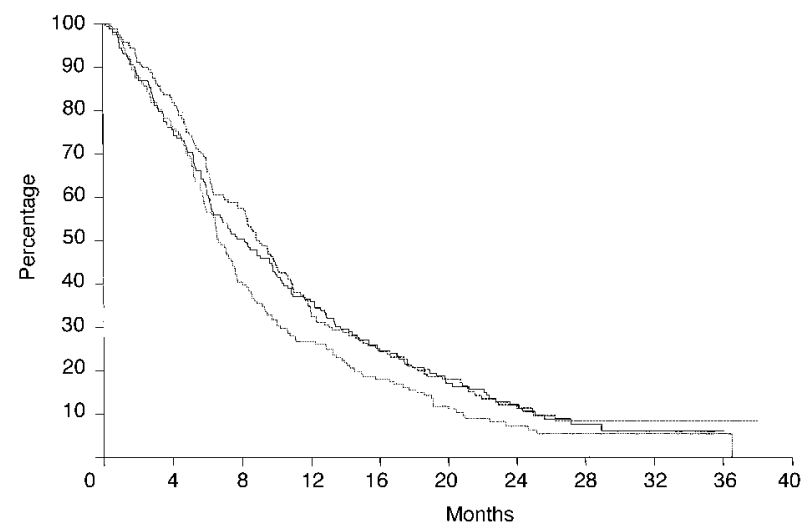

O N Number of patients at risk:

$\begin{array}{lllllllllll}145 & 159 & 120 & 80 & 57 & 39 & 27 & 16 & 5 & 1 & 1\end{array}$ Pacli-CDDP

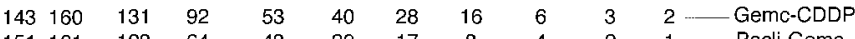

$\begin{array}{lllllllllll}151 & 161 & 123 & 64 & 43 & 29 & 17 & 8 & 4 & 2 & 1\end{array}$

Fig 2. Survival by treatment arm. O, observed; N, number; Pacli, paclitaxel; CDDP, cisplatin; Gemc, gemcitabine.
6 (47 forms received of the 183 forms expected; $25.7 \%$ ) and for assessments during follow-up. This analysis is therefore restricted to the treatment period. There was no significant difference in compliance at the different assessment points between the two experimental arms and the standard arm. When comparing arm B with arm A, no significant difference in global QoL $(P=.816)$ was observed (Fig 4$)$. A statistically $(P<.0001)$ and clinically significant overall improvement was observed for peripheral neuropathy and alopecia in arm B compared with arm A. Nausea and vomiting increased significantly with time but at a similar rate in both arms. Clinically relevant improvement was observed for coughing and insomnia in both arms.

No significant overall treatment effect was found for global QoL $(P=.232)$ comparing arm $\mathrm{C}$ with arm A (Fig 4). There was a difference between the two arms in the way global QoL evolved with time. In arm A, global QoL score increased 10 points between baseline and the end of cycle 2 and thereafter gradually decreased to baseline values at the end of cycle 6 . In arm $\mathrm{C}$, the global QoL score slightly increased between baseline and the end of cycle 1 and then stabilized around baseline value. Nausea and vomiting increased with time in

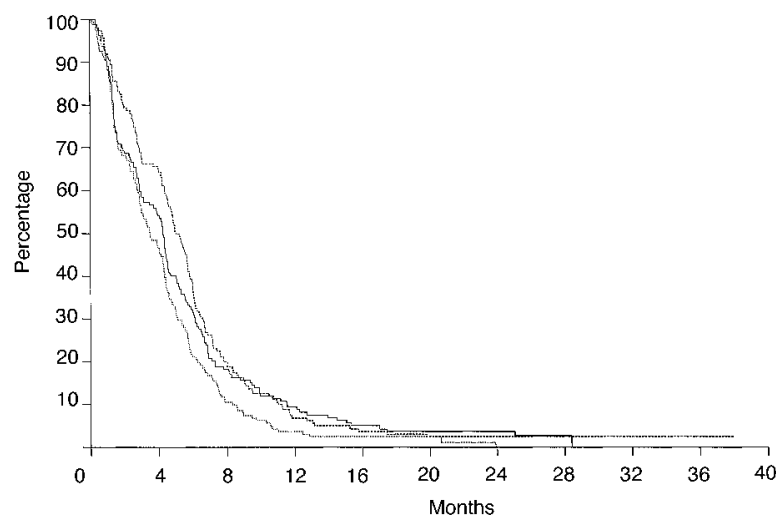

O $\mathrm{N} \quad$ Number of patients at risk:

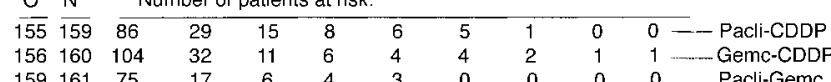

0

Fig 3. Progression-free survival by treatment arm. $\mathrm{O}$, observed; $\mathrm{N}$, number; Pacli, paclitaxel; CDDP, cisplatin; Gemc, gemcitabine. 
Table 5. Grade 3 and 4 Toxicities (NCl-CTC grading system)

\begin{tabular}{|c|c|c|c|c|c|c|c|}
\hline & \multicolumn{2}{|c|}{$\operatorname{Arm} A(n=159)$} & \multicolumn{2}{|c|}{$\operatorname{Arm} B(n=160)$} & \multicolumn{2}{|c|}{$\operatorname{Arm} C(n=161)$} & \multirow[b]{2}{*}{$P$} \\
\hline & No. & $\%$ & No. & $\%$ & No. & $\%$ & \\
\hline \multirow{2}{*}{ Leucocytopenia } & 19 & 11.9 & 43 & 26.9 & 34 & 21.1 & $A \vee B, .001$ \\
\hline & & & & & & & $A \vee C, .035$ \\
\hline \multirow[t]{2}{*}{ Neutropenia } & 54 & 34.0 & 69 & 43.1 & 49 & 30.4 & $A \vee B, .108$ \\
\hline & & & & & & & $A \vee C, .550$ \\
\hline \multirow[t]{2}{*}{ Platelets } & 2 & 1.3 & 58 & 36.3 & 10 & 6.2 & $\mathrm{~A} \vee \mathrm{B},<.001$ \\
\hline & & & & & & & $A \vee C, .035$ \\
\hline \multirow[t]{2}{*}{ Anemia } & 5 & 3.1 & 19 & 11.9 & 6 & 3.7 & $A \vee B, .005$ \\
\hline & & & & & & & $A \vee C, .999$ \\
\hline \multirow[t]{2}{*}{ Febrile neutropenia } & 2 & 1.3 & 4 & 2.5 & 3 & 1.9 & $A \vee B, .685$ \\
\hline & & & & & & & $A \vee C, .999$ \\
\hline \multirow[t]{2}{*}{ Nausea } & 13 & 8.2 & 20 & 12.5 & 10 & 6.2 & $A \vee B, .270$ \\
\hline & & & & & & & $A \vee C, .524$ \\
\hline \multirow[t]{2}{*}{ Vomiting } & 14 & 8.8 & 20 & 12.5 & 9 & 5.6 & $A \vee B, .365$ \\
\hline & & & & & & & $A \vee C, .287$ \\
\hline \multirow[t]{2}{*}{ Lethargy } & 15 & 9.4 & 19 & 11.9 & 18 & 11.2 & $A \vee B, .587$ \\
\hline & & & & & & & $A \vee C, .714$ \\
\hline \multirow[t]{2}{*}{ Dyspnea } & 13 & 8.2 & 17 & 10.6 & 20 & 12.4 & $A \vee B, .566$ \\
\hline & & & & & & & $A \vee C, .427$ \\
\hline \multirow[t]{2}{*}{ Other skin toxicity* } & 17 & 10.7 & 10 & 6.3 & 19 & 11.8 & $A \vee B, .165$ \\
\hline & & & & & & & $A \vee C, .860$ \\
\hline \multirow[t]{2}{*}{ Cancer pain } & 21 & 13.2 & 20 & 12.5 & 22 & 13.7 & $A \vee B, .869$ \\
\hline & & & & & & & $A \vee C, .999$ \\
\hline
\end{tabular}

Abbreviation: NCl-CTC, National Cancer Institute common toxicity criteria.

*Including alopecia.

both arms, but less so in arm $\mathrm{C}$. The difference was statistically significant $(P=.0047)$ but became clinically relevant only at the end of cycle 5 .

\section{Resource Use}

A detailed analysis of resource use data is the subject of a separate manuscript. Here, we report the base case point estimates of the average total cost (ATC) per patient without the correction for censoring, averaged over all randomly assigned patients and broken down into the principal cost categories (Table 6). ATCs are highest in arm C and statistically significantly lower in arm B than in arm A. Costs of hospital admissions for treatment of adverse events and disease symptoms and for second-line therapy were quite similar in the three arms, so the differences in ATC are mainly due to differences in the costs of administration of chemotherapy and of the cytotoxic agents themselves. Treatment $\mathrm{C}$ was the least costly to administer, but the cytotoxic agents cost more than the double of those used for treatment B. The costs of the cytotoxic agents for treatment A were more than $50 \%$ higher than those for treatment $\mathrm{B}$, but this is partially balanced by lower costs of administration of treatment $\mathrm{A}$.

\section{DISCUSSION}

The results of the randomized phase III study presented here show that neither cisplatin-gemcitabine nor paclitaxel-gemcitabine chemotherapy is superior to the cisplatin-paclitaxel combination for patients with advanced NSCLC in regard to overall survival, progression-free survival, and response rate. The median survival obtained in the reference arm, paclitaxel plus
A

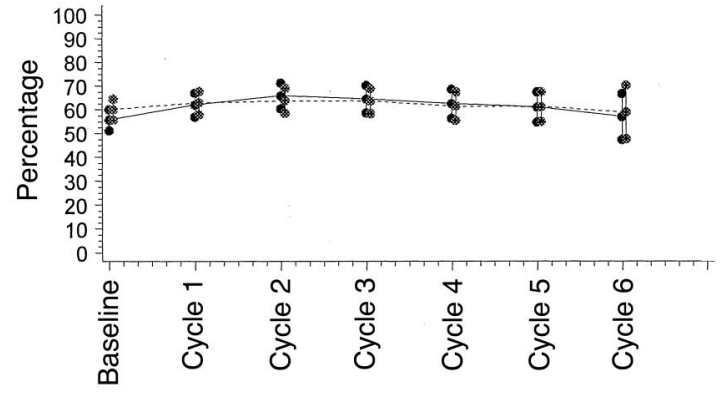

Treatment $\because \bullet$ Pacli-CDDP Gemc-CDDP
B

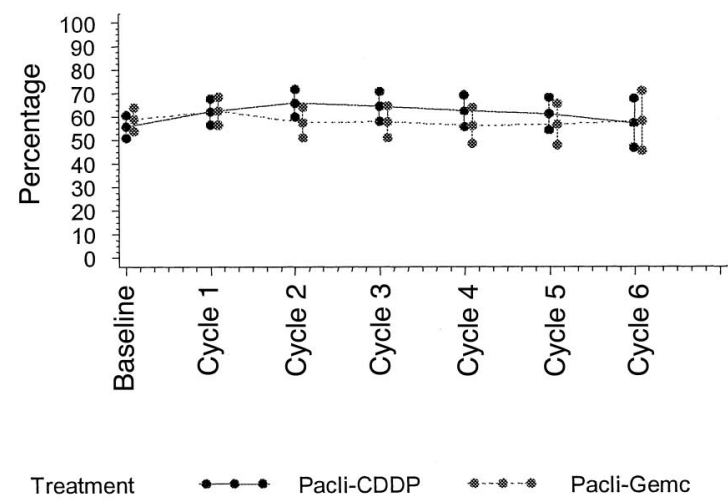

Fig 4. Global quality of life. Arm A versus arm B (A) and arm A versus arm C (B). Pacl, paclitaxel; CDDP, cisplatin; Gemc, gemcitabine. 
Table 6. Summary of Mean Cost Estimates, in Euros, 2002 Values

\begin{tabular}{lrcr}
\hline \multicolumn{1}{c}{ Cost Component } & Arm A & Arm B & Arm C \\
\hline Hospital costs, total & 5,214 & 6,471 & 4,459 \\
Overnight stays & 5,058 & 5,475 & 3,187 \\
Day clinic stays & 156 & 996 & 1,262 \\
Administration of chemotherapy & 2,909 & 4,059 & 2,201 \\
Other reasons & 2,305 & 2.412 & 2,258 \\
Specialist and GP consultations* & 97 & 97 & 97 \\
Cytotoxicst & 8,654 & 5,234 & 11,108 \\
Blood transfusions & 209 & 367 & 140 \\
Second-line therapy & 1,893 & 1,840 & 1,643 \\
ATC per patient & 16,067 & 14,009 & 17,447 \\
\hline
\end{tabular}

Abbreviations: GP, general practitioner; ATC, average total cost; CTG, Dutch National Health Insurance Committee.

${ }^{*}$ Costs calculated assuming a single-visit co-pay (CTG code 010122) because the mean number of specialist consultations is between five and seven in the three groups.

†Chemotherapy includes supportive care (ie, premedication before paclitaxel and antiemetics).

cisplatin, was shorter than that reported in our previous randomized phase III trial, ie, 8.1 months (95\% CI, 6.2 to 9.9 months) versus 9.7 months (95\% CI, 8.2 to 11.9 months) ${ }^{11}$ but comparable to that observed in the reference arm (cisplatin plus paclitaxel) in the recently reported four-arm randomized study of the Eastern Cooperative Oncology Group. ${ }^{17}$ This can be explained by the fact that in the present study, approximately $80 \%$ of the patients entered had stage IV disease, versus $61 \%$ in our previous study. A strong (but according to the predefined statistical criteria, non significant) trend in the nonplatinum arm toward lower overall and progression-free survival was observed as compared with the reference arm. One may question whether the design of the study, which sought to show an improvement in median survival of 50\%, was too optimistic. Indeed, the phase I/II study of the paclitaxel-gemcitabine combination that formed the basis of arm C found a median survival of only 5.4 months (95\% CI, 4.4 to 8.2 months) ${ }^{20}$ On the other hand, to power a three-arm study for a more realistic 30\% increase in median survival would require approximately 1,000 patients to be assigned. Three phase III studies ${ }^{33-35}$ also failed to demonstrate a significant difference in survival between advanced NSCLC patients treated with platinum-based versus nonplatinum-based chemotherapy. In the Italian study, ${ }^{35}$ as in our study, a strong trend for inferior progression-free survival was observed in the nonplatinum arm. However, all these studies, as the study under discussion, were designed to show superiority of the nonplatinum regimens either in regard to survival or response. Equivalence testing of platinum and nonplatinum combinations with the newer agents await adequately powered studies, ${ }^{36}$ ie, studies including much larger numbers of patients.

Multivariate analysis identified PS 2 as the sole factor negatively affecting survival. Retrospective reviews of randomized phase III studies of cisplatin-based chemotherapy have consistently shown that PS 2 patients have a poor survival. ${ }^{8,37-39}$ From a reanalysis of E1594, ${ }^{40}$ it was concluded that the disease process rather than treatment was the cause of the poor overall survival and the observed toxicity in PS 2 patients. Although we did not analyze whether the incidence of grade 3 and 4 toxicities was more prevalent in PS 2 patients, none of the 12 toxic deaths occurred in this patient group.

Because the role of chemotherapy in the treatment of advanced NSCLC is palliative at best, side effects of treatment become a major issue. The side effects of cisplatin limit its use, especially in older patients with concomitant cardiorespiratory disease, and are probably also responsible for the poor acceptance of cisplatin-based chemotherapy in the routine treatment of advanced NSCLC by the medical community. In this study, the only toxicity that differed significantly among the three treatment arms was myelosuppression, being more common in arm B versus arm $\mathrm{A}$, but this did not lead to clinically relevant sequelae. The proportion of patients experiencing grade 3 and 4 toxicities typically associated with the use of cisplatin (eg, nausea and vomiting, renal toxicity, and neurotoxicity) was similar in all arms. This result is at variance with previous studies comparing platinum-based chemotherapy with nonplatinum-based chemotherapy for advanced NSCLC patients. ${ }^{8,33,35,41}$ However, two recent studies also found no difference in toxicity when comparing platinum-treated with nonplatinum-treated patients. ${ }^{34,42}$ QoL analysis during treatment complements the toxicity findings. Global QoL score improved temporarily in all three arms and then returned to baseline values. Negative effects on QoL typically associated with the use of cisplatin, such as nausea and vomiting, were different at a clinically relevant level in the noncisplatin arm only at cycles 5 and 6 and is particularly sensitive to selection bias because of the high number of drop-outs. This is the first study to demonstrate that global QoL in patients with advanced NSCLC is similar for patients treated with platinum-based chemotherapy versus those treated with nonplatinum-based chemotherapy. Several authors advocate short duration of initial chemotherapy (three to four courses) for patients with advanced NSCLC, mainly on the basis of findings of two randomized trials. ${ }^{43,44}$ The median number of courses administered in the two platinum doublets was five. In these arms, survival (8.1 months and 8.9 months) was numerically superior to median survivals as reported by Smith et al (6 months $)^{43}$ and Socinski et al (6.6 months). ${ }^{44}$ Whether the maximum number of courses in the next phase III study should be limited to four or even fewer is still a matter of debate within the EORTC Lung Study Group.

There is increasing pressure to demonstrate the value of new treatments within the health care budget. From the results of the economic evaluation reported here, it may be concluded that the paclitaxel-gemcitabine arm is an inferior option compared with the two cisplatin-containing regimens because the clinical outcomes are no better, both in regard to survival and QoL, whereas the average medical costs are higher. Despite its higher incidence of severe hematologic toxicities and the consequent greater need for blood transfusions, the medical costs of the gemcitabinecisplatin regimen are significantly smaller than those of the paclitaxel-cisplatin regimen. Any medical economic evaluation must by necessity rely on resource unit prices from a particular health care setting and point in time for the determination of direct medical costs engendered by treatment. Because absolute 
and relative unit prices diverge between health care settings in different countries, the generalizability of economical evaluation is a difficult and controversial issue. One would first have to compare unit prices and resource use pattern reported in this evaluation with those prevailing in the health care setting of interest. If both are considered sufficiently similar, the results reported here could reasonably be assumed applicable in a qualitative sense. However, if precise quantitative estimates are needed, an independent economic evaluation should be performed, but available modeling techniques would ease the burden of doing so.

In conclusion, this study demonstrates that the two drug combinations of gemcitabine plus cisplatin and paclitaxel plus gemcitabine do not increase overall survival for the palliative treatment of advanced NSCLC as compared with paclitaxel plus cisplatin. There was a trend toward lower progression-free survival and shorter duration of response for the nonplatinum arm. Treatment was well tolerated and most QoL parameters were equal in all three arms, but the costs associated with the nonplatinum arm were highest. The EORTC Lung Cancer Group will continue to use a platinum-containing regimen as the reference arm in the next randomized study that will be conducted in patients with advanced NSCLC.

\section{ACKNOWLEDGMENT}

We thank Marie-Ange Lentz of the EORTC Data Center for data management, Corneel Coens of the EORTC Data Center for statistical advice on the QoL analysis, and Bristol-Myers Squibb and Eli Lilly for providing paclitaxel and gemcitabine as investigational agents free of charge.

\section{APPENDIX}

The appendix is included in the full-text version of this article, available on-line at www.jco.org. It is not included in the PDF (via Adobe ${ }^{\circledR}$ Acrobat Reader()) version.

\section{AUTHORS' DISCLOSURES OF POTENTIAL CONFLICTS OF INTEREST}

The authors indicated no potential conflicts of interest.

\section{REFERENCES}

1. Parker DM, Whelan SL, Ferlay J, et al: Cancer Incidence in Five Continents. IARC scientific publications, vol 143. Lyon, France, International Agency for Research on Cancer, 1997

2. Page NC, Read WL, Tierney RM, et al: The epidemiology of small cell lung carcinoma. Proc Am Soc Clin Oncol 21:305a, 2002 (abstr 1216)

3. Souquet PJ, Chauvin F, Boissel JP, et al: Polychemotherapy in advanced non small cell lung cancer: A meta analysis. Lancet 342:19-21, 1993

4. Non-Small Cell Lung Cancer Collaborative Group: Chemotherapy in non-small cell lung cancer: A meta-analysis using updated data on individual patients from 52 randomized clinical trials. BMJ 311:899-909, 1995

5. Cullen MH, Billingham LJ, Woodroffe CM, et al: Mitomycin, ifosfamide, and cisplatin in unresectable non-small-cell lung cancer: Effects on survival and quality of life. J Clin Oncol 17:3188-3194, 1999

6. Ranson M, Davidson N, Nicolson M, et al: Randomized trial of paclitaxel plus supportive care versus supportive care for patients with advanced non-small-cell lung cancer. J Natl Cancer Inst 92:1074-1080, 2000

7. Anderson H, Hopwood P, Stephens RJ, et al: Gemcitabine plus best supportive care (BSC) vs BSC in inoperable non-small cell lung cancer: A randomized trial with quality of life as the primary outcome. Br J Cancer 83:447-453, 2000

8. Wozniak AJ, Crowley JJ, Balcerzak SP, et al: Randomized trial comparing cisplatin with cisplatin plus vinorelbine in the treatment of advanced non-smal-cell lung cancer: A Southwest Oncology Group study. J Clin Oncol 16:2459-2465, 1998

9. Sandler AB, Nemunaitis J, Denham C, et al: Phase III trial of gemcitabine plus cisplatin versus cisplatin alone in patients with locally advanced or metastatic non-small-cell lung cancer. J Clin Oncol 18:122-130, 2000

10. Bonomi P, Kim K, Fairclough D, et al: Comparison of survival and quality of life in advanced non-small-cell lung cancer patients treated with two dose levels of paclitaxel combined with cisplatin versus etoposide with cisplatin: Results of an Eastern Cooperative Oncology Group trial. J Clin Oncol 18:623-631, 2000

11. Giaccone G, Splinter TAW, DeBruyne C, et al: Randomized study of paclitaxel-cisplatin versus cisplatin-teniposide in patients with advanced non-small-cell lung cancer. J Clin Oncol 16:2133-2141, 1998

12. Cardenal F, Lopez-Cabrerizo MP, Anton A, et al: Randomized phase III study of gemcitabine-cisplatin versus etoposide-cisplatin in the treatment of locally advanced or metastatic non-small-cell lung cancer. J Clin Oncol 17:12-18, 1999
13. Crino L, Scagliotti GV, Ricci S, et al: Gemcitabine and cisplatin versus mitomycin, ifosfamide, and cisplatin in advanced non-small-cell lung cancer: A randomized phase III study of the Italian Lung Cancer Project. J Clin Oncol 17:3522-3530, 1999

14. Earle CC, Evans WK: Cost-effectiveness of paclitaxel plus cisplatin in advanced non-small-cell lung cancer. Br J Cancer 80:815-820, 1999

15. Lees M, Aristides M, Maniadakis N, et al: Economic evaluation of gemcitabine alone and in combination with cisplatin in the treatment of non small cell lung cancer. Pharmacoeconomics 20:325-337, 2002

16. Annemans L, Giaccone G, Vergenegre A: The cost-effectiveness of paclitaxel $($ Taxol $)+$ cisplatin is similar to that of teniposide + cisplatin in advanced non-small cell lung cancer: A multicountry analysis. Anticancer Drugs 10:605-615, 1999

17. Schiller JH, Harrington D, Belani CP, et al: Comparison of four chemotherapy regimens for advanced non-small-cell lung cancer. N Engl J Med 346:92-98, 2002

18. Leudke DW, Einhorn L, Ommara GA, et al: Randomized comparison of two combination regimens versus minimal chemotherapy in non-smallcell lung cancer: A Southern Cancer Study Group trial. J Clin Oncol 8:886-891, 1990

19. Gridelli C, Perrone F, Palmeri S, et al: Mitomycin plus vindesine plus etoposide (MEV) versus mitomycin plus vindesine plus cisplatin (MVP) in stage IV non-small-cell-lung cancer: A phase III multicentre randomized trial. Ann Oncol 7:821-826, 1996

20. Giaccone G, Smit EF, Van Meerbeeck JPAM, et al: A phase I/II study of gemcitabine and paclitaxel in advanced non-small cell lung cancer patients. Ann Oncol 11:109-112, 2000

21. Mountain CF: Revisions in the international system for staging lung cancer. Chest 111:1710-1717, 1997

22. World Health Organization: Handbook for Reporting Results of Cancer Treatment—Publication no 48. Geneva, Switzerland, World Health Organization, 1979

23. NCIC-CTC grading system. http://ctep.cancer.gov/forms/CTCv20_430-992.pdf

24. Pocock SJ, Simon R: Sequential treatment assignment with balancing for prognostic factors in the controlled clinical trial. Biometrics 31:103-115, 1975

25. George SL: The required size and length of a phase III clinical trial, in Buyse ME, Staquet MJ, Sylvester RJ (eds): Cancer Clinical Trials: Methods and Practice. Oxford, United Kingdom, Oxford Medical Publications, 1998, pp 287-311 
26. Lann KKG, DeMets DL: Discrete sequential boundaries for clinical trials. Biometrica 70:659-663, 1983

27. O’Brian PC, Fleming TR: A multiple testing procedure for clinical trials. Biometrics 35:549-556, 1979

28. Kaplan EL, Meier P: Nonparametric estimation from incomplete observations. J Am Stat Assoc 53:457-481, 1958

29. Mantel N: Evaluation of survival data and two new rank order statistics arising in its consideration. Cancer Chemother Rep 50:163-170, 1966

30. Cox DR: Regression models and life-tables. J R Stat Soc B 34:187202, 1972

31. Fayers P, Aaronson N, Bjordal K, et al: EORTC QLQ Scoring Manual (ed 3). Brussels, Belgium, European Organization for Research and Treatment of Cancer, 2001

32. Osoba D, Rodrigues G, Myles J, et al: Interpreting the significance of changes in health-related quality-of-life scores. J Clin Oncol 16:139-144, 1998

33. Georgoulias V, Papadakis E, Alexopoulos A, et al: Platinum-based and non-platinum-based chemotherapy in advanced non-small-cell lung cancer: A randomised multicentre trial. Lancet 357:1478-1484, 2001

34. Kosmidis PA, Mylonakis N, Nicolaides C, et al: Paclitaxel plus carboplatin versus gemcitabine plus paclitaxel in advanced non-small-cell lung cancer: A phase III randomized trial. J Clin Oncol 20:3578-3585, 2002

35. Gridelli C, Shepherd F, Perrone F, et al: Gemvin III: A phase III study of gemcitabine plus vinorelbine (GV) compared to cisplatin plus vinorelbine or gemcitabine chemotherapy (PCT) for stage IIIb or IV non-small cell lung cancer (NSCLC): An Italo-Canadian study. Proc Am Soc Clin Oncol 21:292a, 2002 (abstr 1165)

36. Van Meerbeeck JP, Legrand C, van Klaveren RJ, et al: Chemotherapy for non-small cell lung cancer. Lancet 358:1271-1272, 2001
37. Paesmans M, Sculier JP, Libert $\mathrm{P}$, et al: Prognostic factors for survival in advanced non-small-cell lung cancer: Univariate and multivariate analyses including recursive partitioning an amalgamation algorithms in 1,052 patients-The European Lung Cancer Working Party. J Clin Oncol 13:12211230, 1995

38. Jiroutek M, Johnson D, Blum R, et al: Prognostic factors in advanced non-small-cell lung cancer (NSCLC): Analysis of Eastern Cooperative Oncology Group trials from 1981-1992. Proc Am Soc Clin Oncol 17:461, 1998 (abstr 1774)

39. Billingham LJ, Cullen MH: The benefits of chemotherapy in patients subgroups with unresectable non-small cell lung cancer. Ann Oncol 12:16711675,2001

40. Sweeney CJ, Zhu J, Sandler AB, et al: Outcome of patients with a performance status of 2 in Eastern Cooperative Oncology Group Study E1594. Cancer 92:2639-2647, 2001

41. Bunn PA, Kelly K: New chemotherapeutic agents prolong survival and improve quality of life in non-small cell lung cancer: A review of the literature and future directions. Clin Cancer Res 4:1087-1100, 1998

42. Yamamoto N, Fukuoka M, Nakagawa K, et al: Randomized phase II study of docetaxel (DOC) plus cisplatin (CDDP) versus DOC plus irinotecan in advanced non-small cell lung cancer (NSCLC): A West-Japan Thoracic Oncology Group (WJTOG) study. Ann Oncol 11:S107, 2000 (abstr)

43. Smith IE, O'Brian MER, Talbot DC, et al: Duration of chemotherapy in advanced non-small-cell lung cancer: A randomized trial of three versus six courses of mitomycin, vinblastine, and cisplatin. J Clin Oncol 19:13361343,2001

44. Socinski MA, Schell MJ, Peterman A, et al: Phase III trial comparing a defined duration of therapy versus continuous therapy followed by second-line therapy in advanced stage IIIB/IV non-small-cell lung cancer. J Clin Oncol 20:1335-1343, 2002 